Int. J. Odontostomat., 8(3):475-480, 2014.

\title{
Obesidad y Caries Dental Problemas de Salud Pública en una Población Escolar
}

\author{
Obesity and Dental Caries Public Health Problems in a School Population
}

\author{
María del Pilar Adriano Anaya*; Tomás Caudillo Joya*; \\ María Lilia Adriana Juárez López* \& Pilar Alejandra Caudillo Adriano**
}

\begin{abstract}
ADRIANO, A. M. P.; CAUDILLO, J. T.; JUÁREZ, L. M. L. A.\& CAUDILLO, A. P. A. Obesidad y caries dental problemas de salud pública en una población escolar. Int. J. Odontostomat., 8(3):475-480, 2014.

RESUMEN: En diversos estudios se ha planteado a la obesidad como un factor de riesgo para el desarrollo de la caries dental, sin embargo la literatura ha demostrado resultados discordantes, por lo que es necesario seguir investigando sobre la asociación de éstos dos problemas que son considerados de salud pública. Identificar la asociación entre el Índice de Masa Corporal (IMC) y la caries dental de la población escolar de seis a doce años de 19 escuelas primarias públicas localizadas en los doce pueblos en que se organiza la delegación Milpa Alta, en el Distrito Federal (México). El estudio fue descriptivo, comparativo y transversal, con una muestra por conveniencia de 4734 escolares que presentaron la autorización por escrito, previo consentimiento informado de los padres o tutores. El sobrepeso se midió con el IMC y la caries dental con el indicador CPOD. La información se capturó en el Programa de Cómputo SPSS Versión 15.0. Del total de escolares el 9,3\% se ubicó en el percentil de bajo peso con un promedio de dientes cariados de 1,9. El 50,4\% dentro del normal con 2,5 dientes cariados, el $19,6 \%$ con sobrepeso y con 2,6 dientes con historia de caries dental, el $11,1 \%$ con obesidad y 3,2 dientes cariados y el 9,5\% con obesidad endógena y 2,9 dientes con caries dental. Es importante estudiar la posible asociación entre la caries dental y la obesidad ya que tienen un riesgo en común, la nutrición.
\end{abstract}

PALABRAS CLAVE: obesidad, sobrepeso, caries dental, índice de masa corporal.

\section{INTRODUCCIÓN}

La caries dental y la obesidad son problemas de salud con alta prevalencia en la población escolar y se encuentran influenciadas por la ingesta de alimentos con alto contenido de azúcar.

Según la Organización Mundial de la Salud (OMS), en las conclusiones del Informe Mundial Sobre Salud Bucodental (OMS, 2004), se estima que en el planeta cinco mil millones de personas han sufrido caries dental. Con respecto a la obesidad, un reporte recientemente publicado por la Organización de Naciones Unidas para la Alimentación y la Agricultura (FAO), México es el país con mayor porcentaje de población con obesidad en el mundo (CNN México, 2014). La Encuesta Nacional de Salud y Nutrición 2012 encontró que los niños en edad escolar de 5 a 11 años presentaron una prevalencia nacional combinada de sobrepeso y obesidad de $34,4 \%, 19,8 \%$ para sobrepeso y $14,6 \%$ para obesidad (Instituto Nacional de Salud Pública, 2012).

Godlewski et al. (2008), señala que la obesidad ha progresado tanto en niños como en adultos, y que se asocia con otras patologías como la caries dental. De igual manera, argumenta que la obesidad y las patologías orales tienen factores de riesgo comunes, y uno en particular es la dieta.

Kopycka-Kedzierawski et al. (2008) en estudios semejantes, manifiestan el comportamiento de la experiencia de caries en distintos grupos de edades de niños, relacionado con el peso, y concluyen que los niños con peso normal están menos afectados por caries que los obesos.

\footnotetext{
* Facultad de Estudios Superiores Zaragoza, Universidad Nacional Autónoma de México, México D.F., México.

"Alumna, Universidad Autónoma Metropolitana, Xochimilco, México.
} 
Un estudio realizado por Martínez Sotolongo \& Martínez Brito (2010), concluyó que los mayores valores de caries dental correspondieron a los obesos $(24 \%)$, mientras que el $6,9 \%$ perteneció a los normopesos. Se comprobó la existencia de diferencias estadísticas significativas entre los normopesos y obesos afectados por caries, con un valor de $p<0,01$.

Otro estudio realizado en México en población escolar de 11 a 15 años, se encontró que en conjunto, el número de dientes cariados y la presencia de higiene oral son estadísticamente significativas con relación al Índice de Masa Corporal (IMC) por edad y sexo en escolares. Lo que sustenta la hipótesis de que a mayor grado de peso en el escolar, expresada ésta en términos de morbilidad, es mayor la probabilidad de que se presenten dientes cariados o con higiene oral deficiente (Zelocuatecatl et al., 2005).

Es importante seguir estudiando la posible asociación entre la caries dental y la obesidad ya que son problemas de salud pública en el mundo, hasta el momento existen contradicciones en los resultados de los estudios que se han realizado sobre esta posible asociación. Es por ello que presentamos los resultados de esta investigación con el propósito de identificar la asociación de la obesidad y la caries dental, en la población escolar de seis a doce años en la delegación Milpa Alta, ubicada en la zona sur del Distrito Federal.

Esta delegación, se encuentra organizada en doce pueblos: Villa Milpa Alta, San Pedro Atocpan, San Antonio Tecomitl, San Lorenzo Tlacoyucan, San Salvador Cuauhtenco, San Jerónimo Miacantla, San Agustín Ohtenco, San Pedro Oztotepec, San Francisco Tecoxpa, San Juan Tepejahuac, Santa Ana Tlacotenco, y San Bartolomé Xicomulco. Los tres primeros ubicados en la zona urbana y el resto en la rural (INEGI, 2000; INEGI, 2010; Delegación Milpa Alta, 2006).

\section{MATERIAL Y MÉTODO}

Este proyecto de investigación se sometió para su evaluación a la Unidad de Investigación y Posgrado de la FES-Zaragoza para su autorización y forma parte de la Línea de Investigación Salud Pública en Estomatología. El estudio fue de tipo descriptivo, comparativo, transversal y observacional y se llevó a cabo en 4734 escolares que entregaron su permiso firma- do previo consentimientoinformado por parte de los padres o tutores. Se estandarizó a 10 pasantes de la Carrera de Cirujano Dentista en el manejo de la Antropometría (peso y talla, Índice de Masa Corporal), siguiendo el método de Habitch (de Onis \& Habitch, 1996; Habitch et al., 1974; Habitch, 1974).

Para obtener la talla de los escolares se utilizó un estadiómetro marca SECA de $200 \mathrm{~cm}$. Para el peso una báscula digital de cristal templado de $182 \mathrm{Kg}$ de capacidad. Una vez pesados y medidos los escolares se obtenía el IMC, que se define como una medida de asociación entre el peso y la talla de un individuo. Ideado por el estadístico belga L. A. J. Quetelet, por lo que también se conoce como índice de Quetelet (Rodríguez et al., 2010). El resultado se registró en los gráficos del Centro de Estadística de Salud.

Para la clasificación de sobrepeso existen varias tablas de referencias que tienen valores de mediana diferentes y por lo tanto un niño con un valor para el IMC puede ser clasificado como normal para una tabla de referencia y para otra puede ser con sobrepeso. Los valores de referencia para éste estudio son los de la Asociación Latinoamericana de Diabetes ALADE (Tabla I) (Asociación Latinoamericana de Diabetes, 2014; Rosas Guzman, 2009).

Tabla I. Clasificación del Índice de Masa Corporal Asociación Latinoamericana de Diabetes.

\begin{tabular}{lcc}
\hline Clasificación & $\mathbf{I M C}\left(\mathbf{K g} / \mathbf{m}^{\mathbf{}}\right)$ & Riesgo \\
\hline Peso bajo & $<\mathrm{p} 10$ & Bajo \\
Normal & $<\mathrm{p} 10-85$ & Promedio \\
Sobrepeso & $\geq \mathrm{p} 85$ & Moderado \\
Obesidad & $\geq 95$ & Alto \\
Obesidad mórbida & $\geq 97$ & Muy alto \\
\hline
\end{tabular}

Para obtener la prevalencia de caries dental (CPOD), se llevó a cabo la calibración (concordancia intraexaminador, interexaminador de más del $85 \%$ ), seleccionando de los 10 pasantes a cinco revisores que alcanzaron dicha prueba, siguiendo los criterios propuestos por la OMS (OMS, 1997).

Para calcular el Índice de Caries Significativa (ISC) (Piovano et al., 2010), se dividió a toda la población estudiada en tres tercios tomando para su estudio al CPOD del último tercio que es donde se encuentra la población más afectada por caries dental, por lo que sus resultados presentan mayor realidad de este problema de esta población. 
El Índice de Necesidades de Tratamiento (INT) (Piovano et al.), de los escolares de las siete primarias se obtuvo aplicando la siguiente fórmula:

INT= (Promedio de dientes cariados/( Promedio de dientes cariados + promedio dientes obturados) $) \times 100$

La exploración oral se realizó con luz natural con las barreras de protección propuestas por la Norma oficial Mexicana para el control y manejo de infecciones orales (NORMA Oficial Mexicana NOM-087ECOL-SSA1-2002, 2014).

La información se capturó en el Programa de cómputo SPSS versión 15.0 se obtuvieron: Estadísticas descriptivas como es el promedio de caries dental, porcentaje de IMC, ANOVA (Bonferroni), para IMC y caries dental, $t$ de Student para significancia de caries dental y sexo y $\mathrm{X}^{2}$ de Pearson para significancia entre caries dental y edad.

\section{RESULTADOS}

De los 4734 escolares participantes 2148 pertenecen al sexo masculino y 2586 al femenino, 2213 viven en la zona urbana y 2521 en la rural.

Con respecto a la caries dental en dentición permanente aumentó conforme a la edad observando 0,9 dientes cariados a los seis años y 4,4 a los doce. Encontrando significancia estadística X2 $=204,800$ $\mathrm{p}=0,000$ (Tabla II).

La caries dental tuvo un comportamiento similar tanto en el sexo femenino $(2,6)$, como masculino $(2,5)$. No encontrando significancia estadística, $\mathrm{t}=3,093, \mathrm{p}=0,79$ (Tabla III).

De las 19 primarias participantes, ocho de ellas se encuentran en la zona urbana y 11 en la rural, el promedio de caries dental global fue de 2,6 dientes con historia de la enfermedad, encontrando significancia estadística. ANOVA=16,492 p=0,000 (Tabla IV).

Al describir el Índice de Masa Corporal, el 9,3\% de los escolares se encontraron con bajo peso, el $50,4 \%$ normales, $19,6 \%$ con sobrepeso, el $11,1 \%$ con obesidad y el $9,5 \%$ presentaron obesidad endógena. Los promedios de caries dental (CPOD), más elevados 3,2 dientes cariados, se presentaron en los esco-
Tabla II. Distribución de la caries dental por edad de los escolares.

\begin{tabular}{lcc}
\hline Edad & CPOD & $\mathbf{n}$ \\
\hline 6 años & $0.9 \pm 1.3$ & 590 \\
7 años & $1.6 \pm 1.6$ & 723 \\
8 años & $2.3 \pm 1.6$ & 650 \\
9 años & $2.6 \pm 2.0$ & 811 \\
10 años & $3.2 \pm 2.0$ & 787 \\
11 años & $3.6 \pm 2.5$ & 768 \\
12 años & $4.4 \pm 2.7$ & 405 \\
Total & $2.6 \pm 2.2$ & 4734 \\
& \multicolumn{2}{c}{$\mathrm{X}^{\overline{2}} \mathrm{Z}_{04}$} \\
\hline
\end{tabular}

CPOD=Dientes Cariados, Perdidos, Obturados.

Tabla III. Distribución de la caries dental por sexo de los escolares.

\begin{tabular}{lcc}
\hline Sexo & CPOD & $\mathbf{n}$ \\
\hline Masculino & $2.5 \pm 2.2$ & 2148 \\
Femenino & $2.6 \pm 2.2$ & 2586 \\
Total & $2.6 \pm 2.2$ & 4734 \\
& $\mathrm{t}=3.093, \mathrm{p}=0.79$ \\
\hline
\end{tabular}

CPOD=Dientes Cariados, Perdidos, Obturados.

lares con obesidad, mientras que los más bajos en los de bajo peso, con 1,9 dientes con historia de la enfermedad. Encontrando significancia estadística $X^{2}=102$, $\mathrm{p}=0,000$ (Tabla V).

Al agrupar a las primarias por zona rural y urbana, el promedio de caries dental en la primera fue de 3,1 dientes con historia de caries dental mientras que en las segunda de 2,1 , con respecto a la prueba $X^{2}=55,211, p=0,000$, hay significancia estadística (Tabla V).

Con respecto al Índice de Caries Significativa global (ISC), el más bajo se observó en los escolares con bajo peso $(4,6)$, y el más elevado en los obesos con 5,4 dientes con historia de esta enfermedad, no hay diferencias estadísticas. $\mathrm{X} 2=52, \mathrm{p}=0,047$ (Tabla V).

El INT, nos indicó que más del $94 \%$ de esta población tiene algún problema oral que tiene que ser tratado (Tabla V).

En la zona urbana se observó más escolares con bajo peso $(67 \%)$, con un promedio de caries dental de 1,5 dientes afectados, que en la rural (33\%), y 2,7 dientes con caries. 
Tabla IV. Primarias por zona, pueblo y distribución de la caries dental de los escolares.

\begin{tabular}{llcccc}
\hline Primaria & Pueblo & Zona & $\mathbf{n}$ & $\%$ & CPOD \\
\hline 1.-Cultura Azteca & Villa Milpa Alta & Urbana & 212 & 4,5 & $1,9 \pm 2,1$ \\
2.-Cultura Azteca Vespertino & Villa Milpa Alta & Urbana & 274 & 5,8 & $2,0 \pm 2,3$ \\
3.-Morelos y Pavón & Villa Milpa Alta & Urbana & 205 & 4,3 & $2,4 \pm 2,0$ \\
4.-Morelos y Pavón Vespertino & Villa Milpa Alta & Urbana & 296 & 6,3 & $2,1 \pm 2,6$ \\
5.-Álvaro Obregón & San Pedro & Urbana & 344 & 7,3 & $2,0 \pm 2,0$ \\
6.-Rosa Villanueva & San Antonio & Urbana & 263 & 5,6 & $1,6 \pm 1,8$ \\
7.-Legorreta & San Antonio & Urbana & 259 & 5,5 & $2,9 \pm 2,4$ \\
8.-Bonfil & San Antonio & Urbana & 360 & 7,6 & $3,2 \pm 2,0$ \\
9.-Tláloc & San Agustín & Rural & 224 & 4,7 & $2,4 \pm 2,5$ \\
10.-Francisco del Olmo & Santa Ana & Rural & 317 & 6,7 & $1,8 \pm 2,1$ \\
11.-Francisco del Olmo Vespertino & Santa Ana & Rural & 134 & 2,8 & $2,6 \pm 2,1$ \\
12.-Miguel Alemán & San Salvador & Rural & 86 & 1,8 & $3,1 \pm 1,9$ \\
13.-Progreso campesino & San Lorenzo & Rural & 279 & 5,9 & $3,0 \pm 2,7$ \\
14.-Sierra Leona & San Jerónimo & Rural & 210 & 4,4 & $3,2 \pm 2,3$ \\
15.-Benito Juárez & San Bartolomé & Rural & 270 & 5,7 & $3,0 \pm 2,1$ \\
16.-Miguel Hidalgo & San Agustín & Rural & 432 & 9,1 & $3,2 \pm 2,0$ \\
17.-Plan sexenal & San Pablo & Rural & 130 & 2,7 & $3,0 \pm 2,4$ \\
18.-Teutli & San Francisco & Rural & 265 & 5,6 & $2,9 \pm 2,0$ \\
19.-Loyola & San Juan & Rural & 174 & 3,7 & $3,1 \pm 1,9$ \\
Total & & & 4734 & 100,0 & $2,6 \pm 2,2$ \\
& & & ANOVA=16.492, p=0.000
\end{tabular}

CPOD=Dientes Cariados, Perdidos, Obturados.

Tabla V. Distribución del IMC por Indicadores de caries dental de los escolares.

\begin{tabular}{|c|c|c|c|c|c|c|c|c|c|c|c|}
\hline \multirow{2}{*}{ IMC } & \multicolumn{5}{|c|}{ Globales } & \multicolumn{3}{|c|}{ Rural } & \multicolumn{3}{|c|}{ Urbana } \\
\hline & $\mathbf{n}$ & $\%$ & CPOD & ISC & INT & $\mathbf{n}$ & $\%$ & CPOD & $\mathbf{n}$ & $\%$ & CPOD \\
\hline Bajo peso & 440 & 9,3 & $1,9 \pm 1,8$ & 4,6 & $94 \%$ & 146 & 33 & $2,7 \pm 1,9$ & 294 & 67 & $1,5 \pm 1,7$ \\
\hline Normal & 2386 & 50,4 & $2,5 \pm 2,2$ & 5,1 & $99 \%$ & 1206 & 51 & $2,9 \pm 2,1$ & 1180 & 49 & $2,1 \pm 2,2$ \\
\hline Sobrepeso & 929 & 19,6 & $2,6 \pm 2,0$ & 4,7 & $97 \%$ & 522 & 53 & $3,0 \pm 2,0$ & 407 & 47 & $2,0 \pm 2,0$ \\
\hline Obesidad & 527 & 11,1 & $3,2 \pm 2,5$ & 5,4 & $98 \%$ & 273 & 52 & $3,5 \pm 2,6$ & 254 & 48 & $2,7 \pm 2,3$ \\
\hline Obesidad Endógena & 452 & 9,5 & $2,9 \pm 2,4$ & 5,2 & $98 \%$ & 282 & 62 & $3,3 \pm 2,3$ & 170 & 38 & $2,2 \pm 2,3$ \\
\hline \multirow[t]{2}{*}{ Total } & 4734 & 100,0 & $2,6 \pm 2,2$ & 5,0 & $98 \%$ & 2429 & 51,3 & $3,1 \pm 2,2$ & 2305 & 48,7 & $2,1 \pm 2,3$ \\
\hline & \multicolumn{3}{|c|}{$X_{2}=102, p=0,000$} & $x^{2=} 52, p=0,047$ & & & & \multicolumn{3}{|c|}{$X_{2}=55,211, p=0,000$} & \\
\hline
\end{tabular}

IMC= Índice de Masa Corporal; CPOD=Dientes Cariados, Perdidos, Obturados; ISC= índice Significante de Caries Dental; INT=Índice de Necesidades de Tratamiento.

Se encontraron más obesos endógenos (62\%) en la zona rural, con 3,3 dientes cariados, que en la urbana (38\%) y 2,2 dientes con caries (Tabla V). Con respecto al sexo se encontró que hay más niñas con bajo peso (227) con 2,0 dientes cariados, con sobrepeso (493) con 2,6 y con obesidad (298) y 3,2 dientes con caries dental. Se encontraron a más niños con obesidad endógena (251) con 2,7 dientes cariados. Dentro del rubro de normalidad hay 1367 niñas y 1019 niños. Con 2,6 y 2,5 dientes con historia de caries dental. Encontrando significancia estadística, X2=4733, $p=0,000$ (Tabla VI).
Tabla VI. Distribución del IMC por sexo y promedio de caries dental.

\begin{tabular}{lcccc}
\hline \multirow{2}{*}{ IMC } & \multicolumn{2}{c}{ Masculino } & \multicolumn{2}{c}{ Femenino } \\
\cline { 2 - 5 } & CPOD & $\mathbf{n}$ & CPOD & $\mathbf{n}$ \\
\hline Bajo peso & $1,8 \pm 1,8$ & 213 & $2,0 \pm 1,9$ & 227 \\
Normal & $2,5 \pm 2,3$ & 1019 & $2,6 \pm 2,2$ & 1367 \\
Sobrepeso & $2,6 \pm 2,1$ & 436 & $2,6 \pm 2,0$ & 493 \\
Obeso & $3,1 \pm 2,5$ & 229 & $3,2 \pm 2,6$ & 298 \\
Obeso endógeno & $2,7 \pm 2,4$ & 251 & $3,1 \pm 2,4$ & 201 \\
Total & $2,5 \pm 2,2$ & 2148 & $2,6 \pm 2,2$ & 2586 \\
& & & $X^{L}=4733, p=0,000$
\end{tabular}

IMC= Índice de Masa Corporal; CPOD=Dientes Cariados, Perdidos, Obturados. 


\section{DISCUSIÓN}

El objetivo de éste estudio fue el Identificar la asociación entre el Índice de Masa Corporal y la caries dental de la población escolar de seis a doce años de 19 primarias públicas localizadas en los doce pueblos en que se organiza la delegación Milpa Alta, considerada con mayor pobreza y marginación en el Distrito Federal. Para esta muestra de 4734 escolares si se encontró dicha asociación ya que los promedios de caries dental (CPOD), más elevados 3,2 dientes cariados, se presentaron en los escolares con obesidad, mientras que los menores, en los de bajo peso, con 1,9 dientes con historia de la enfermedad.

Hasta la fecha, no se conoce si realmente existe una asociación entre la caries dental y la obesidad, Pannunzio et al. (2010), afirmaron que niños con mayor IMC, presentaron alteraciones en la composición de la saliva (concentración de fosfatos, ácido salicílico libre, proteínas y actividad de la peroxidasa) como factores favorables para condicionar caries. Por otro lado la alta ingesta de carbohidratos, su permanencia en la boca y la falta del cepillado dental es otra de las causas de la obesidad y de la caries dental. Sin embargo los estudios que se han realizado, sus resultados son heterogéneos ya que algunos han encontrado esta asociación y otros no, por lo que es necesario seguir investigando sobre este tema.

Godlewski (2008) en sus estudios asocia estos dos problemas, así como Kopycka-Kedzierawski et al. Un estudio en Cuba (Martínez Sotolongo \& Martínez Brito), como el realizado en México (Zelocuatecatl et al., 2005), también encontraron dicha asociación.

Con respecto al Índice de Caries Dental Significativo y el IMC de los escolares, los promedios estuvieron muy por encima de lo esperado por la OMS para el año 2015 donde este indicador se debe encontrar en 3,0 dientes cariados, En este estudio el global fue de 5,0 dientes con historia de la enfermedad.

Las necesidades de tratamiento indican que el $94 \%$ de los escolares tiene alguna necesidad en la cavidad oral que no ha sido atendida por la Práctica Profesional, lo que demuestra la baja cobertura en este grupo de población ya sea la asistencial o privada. Si a esto le sumamos los problemas de bajo peso, sobrepeso, obesidad y obesidad endógena en estos escolares podemos observar su complejidad.
Los resultados que nos arroja este estudio, tomando en cuenta las variables seleccionadas, en las cuales se encontró la asociación entre la caries dental y el IMC, son una muestra de que es necesario el cambio de paradigmas unidimensionales, en donde prevalece la certeza por otros en donde se reconozca la multidimensionalidad de los problemas, la incertidumbre que envuelve a estos, el integrar lo biológico con lo social de tal manera que podamos observar como todos los aspectos se encuentran unidos, en donde se hace necesario el construir puentes lo cuales se encuentran unidos como una red en donde se pueda observar su unidad y en consecuencia comprender dichos problemas y poder generar propuestas que permitan su transformación.

\section{CONCLUSIÓN}

Los resultados que se obtuvieron en este estudio se pudo observar la asociación entre la caries dental y el índice de masa corporal, ya que el promedio de caries dental más elevado, lo presentaron los escolares con sobre peso y los obesos. Dicha asociación nos permite observar la multidimensionalidad en su determinación por lo que habrá que proponer paradigmas alternativos como es el del Pensamiento Complejo de tal manera que nos permita la integración entre lo biológico y lo social para su comprensión y proponer alternativas de solución.

Es importante trabajar en conjunto con los padres de familia y autoridades educativas, ya que el refrigerio que se vende en las primarias debe considerarse muy importante por el valor nutritivo que debe contener, un porcentaje muy elevado de los escolares desayuna a la hora del recreo ya que asisten a la escuela sin haberlo hecho en su casa.

Es relevante el seguir realizando este tipo de estudios ya que la obesidad puede ser un factor de riesgo para el desarrollo de la caries dental.

ADRIANO, A. M. P.; CAUDILLO, J. T.; JUÁREZ, L. M. L. A.\& CAUDILLO, A. P. A. Obesity and dental caries public health problems in a school population. Int. J. Odontostomat., 8(3):475-480, 2014.

ABSTRACT: Several studies have proposed obesity as a risk factor for the development of dental caries, but the literature has shown conflicting results, so further research is needed on the association of these two problems which are considered health public. The objective of this study was to identify the association between body mass index (BMI) and dental caries in schoolchildren aged six 
to twelve years of 19 public elementary schools located in the twelve villages in the Milpa Alta delegation is organized in the Federal District (Mexico). The study was descriptive, comparative and transversal, with a convenience sample of 4734 students who took the written consent, informed consent from parents or guardians. Overweight was measured using the $\mathrm{BMI}$ and dental caries CPOD indicator. The information is captured in the Software SPSS version 15.0. Of the total $9.3 \%$ of school started off low weight percentile with an average of 1.9 decayed teeth. $50.4 \%$ within the normal with 2.5 decayed teeth, $19.6 \%$ overweight and 2.6 teeth with a history of dental caries, $11.1 \%$ obese and 3.2 decayed teeth and $9.5 \%$ with endogenous obesity and 2.9 teeth with dental caries. It is important to study the possible association between dental caries and obesity as they have a common risk, nutrition.

KEY WORDS: obesity, overweight, dental caries, body mass index.

\section{REFERENCIAS}

Asociación Latinoamericana de Diabetes (ALAD). Guía ALAD de "Diagnóstico, control, prevención y tratamiento del Síndrome Metabólico en pediatría". 2014. Disponible en: http://www.aladI a t i no a merica.org/DOCConsenso/ SX\%20METABOLICO\%20EN\%20PEDIATRIA.pdf

CNN México. Obesidad: el enemigo público. La historia de cómo kilo a kilo México se volvió líder en obesidad. 2013. Disponible en: http://mexico.cnn.com/salud/2013/07/12/la-historia-decomo-kilo-tras-kilo-mexico-se-volvio-lider-en-obesidad

Delegación Milpa Alta. Dirección General de Desarrollo Social, Subdirección de Cultura y Recreación, San Bartolomé Tepenahuac, México, 2006.

de Onis, M. \& Habitch, J. P. Anthropometric reference data for international use: recommendations from a World Health Organization Expert Committee. Am. J. Clin. Nutr., 64(4):6508, 1996.

Godlewski, A. E.; Veyrune, J. L. \& Nicolas, E. Obesity and oral health: risk factors of obese patients in dental practice. Odontostomatol. Trop., 31(123):25-32, 2008.

Habitch, J. P. Standardization of quantitative epidemiological methods in the field. Bol. Oficina Sanit. Panam., 76(5):375-84, 1974.

Habitch, J. P.; Martorell, R.; Yarbrough, C.; Malina, R. M. \& Klein, R. E. Height and weight standards for preschool children. How relevant are ethnic differences in growth potential? Lancet, 1(7858):611-4, 1974.

Instituto Nacional Estadística Geografía e Informática (INEGI). Marco Geoestadístico. México D. F., Instituto Nacional Estadística Geografía e Informática (INEGI), 2000.

Instituto Nacional Estadística Geografía e Informática (INEGI). Censo México 2010. México D. F., Instituto Nacional Estadística Geografía e Informática (INEGI), 2010.

Instituto Nacional de Salud Pública. Encuesta Nacional de salud y nutrición 2012. México D. F., Instituto Nacional de Salud Pública, 2012. pp.35. Disponible en: http://ensanut.insp.mx/doctos/ ENSANUT2012_Nutricion.pdf
Kopycka-Kedzierawski, D. T.; Auinger, P.; Billings, R. J. \& Weitzman, M. Caries status and overweight in 2- to 18-year-old US children: findings from national surveys. Community Dent. Oral Epidemiol., 36(2):157-67, 2008.

Martínez Sotolongo, B. \& Martínez Brito, I. Comportamiento de la caries dental en escolares obesos y normopesos de 8 a 13 años. Rev. Med. Electrón, 32(3), 2010.

NORMA Oficial Mexicana NOM-087-ECOL-SSA1-2002. Protección ambiental-Salud ambiental-Residuos peligrosos biológico-infecciosos-Clasificación y especificaciones de manejo. México D. F., Estados Unidos Mexicanos, 2003. Disponible en: http:// www.salud.gob.mx/unidades/cdi/nom/087ecolssa.html

Organización Mundial de la Salud (OMS). Encuestas de salud bucodental. Métodos básicos. $4^{\mathrm{a}}$ ed. Ginebra, Organización Mundial de la Salud (OMS), 1997. pp.33-7.

Organización Mundial de la Salud (OMS). Informe sobre el problema mundial de las enfermedades bucodentales. Gac. Dent., 2004:147, 2004.

Pannunzio, E.; Amancio, O. M.; Vitalle, M. S.; Souza, D. N.; Mendes, F. M. \& Nicolau, J. Analysis of the stimulated whole saliva in overweight and obese school children. Rev. Assoc. Med. Bras., 56(1):32-6, 2010

Piovano, S.; Squassi, A. \& Bordoni, N. Estado del arte de indicadores para la medición de caries dental. Rev. Fac. Odontol. (UBA), 25(58):30-8, 2010.

Rodríguez, G.; Moreno, L. \& Sarría A. La obesidad en la Historia. Sobre el índice de Quetelet y obesidad. Rev. Esp. Obesidad, 8(1):34-40, 2010

Rosas Guzman, J.; Torres Tamayo, M.; Calzada León, R.; Sinay, I.; Costa Gil, J.; de Loredo, L.; Ramos, O.; Lúquez, H.; Lyra da Silva Filho, R.; Cavalcanti da Silva, S.; Ferreira, S.; Tambascia, M.; Godoy, A.; Duarte, E.; Ascher, P.; Carrasco Piña, E.; García Bruce, H.; Pasquel, M.; Villatoro, A.; González Chávez, A.; Suverza Fernández, A.; Lavalle González, F.; Castro Martínez, G.; Alvarado Ruíz, R.; Zúñiga Guajardo, S.; Castillo, R.; Fernández Barros, C.; Sienra, J. C.; Arechavaleta Granel, R.; Perez Sanchez, P.; Romero. A.; Escalante Herrera, A.; Gómez Cruz, J.; Alexanderson, G.; García Rubi, E.; Calzada Leon, R.; Laviada, H.; Solis, E.; Violante, R.; Bastarrachea, R.; Abouganem, D.; Caballero, R.; Seclen Santiesteban, S.; Escaño, F.; Reynoso Rivera, J.; Quesada Giraudo, S.; Brajkovich, I. \& Blanco, M. Guía Alad. Rev. Asoc. Latinoam. Diabetes, 17(1):16-31, 2009.

Zelocuatecatl, A. A.; Ortega, M. M. \& de la Fuente, H. J. Asociación entre el índice de masa corporal y las condiciones bucales en escolares. Rev. Odontol. Mex., 9(4):185-90, 2005.

Dirección para Correspondencia:

María del Pilar Adriano Anaya

Facultad de Estudios Superiores Zaragoza Universidad Nacional Autónoma de México

México D.F.

Recibido : 30-07-2014

MÉXICO

Aceptado: 11-10-2014 